\title{
Standard Redox Potentials of Fe(III) Aqua Complexes \\ Included Into the Cavities of Cucurbit[n]urils $(n=6-8)$ : A DFT Forecast
}

\author{
A.N. Masliy", T.N. Grishaeva, A.M. Kuznetsov \\ Department of Inorganic Chemistry, Kazan National Research Technological University, \\ 420015, Kazan, K. Marx Street 68, Russian Federation \\ e-mail: masliy@kstu.ru
}

Supporting Information 
Table S1: Calculated average $\mathrm{r}\left(\mathrm{M}-\mathrm{OH}_{2}\right)$ bond lengths in $\left[\mathrm{M}\left(\mathrm{H}_{2} \mathrm{O}\right)_{6} \cdot 12 \mathrm{H}_{2} \mathrm{O}\right]^{3+2+}$ aqua complexes in comparison with experimental data

\begin{tabular}{|c|c|c|c|c|}
\hline \multirow{2}{*}{$\mathrm{M}$} & \multicolumn{4}{|c|}{$\mathrm{R}\left(\mathrm{M}-\mathrm{OH}_{2}\right), \AA$} \\
\cline { 2 - 5 } & $\mathrm{C}$ & \multicolumn{2}{c|}{$\mathrm{M}^{2+}$} \\
\cline { 2 - 5 } & $\mathrm{Calc}$ & $\mathrm{Exp}^{3+}$ & $\mathrm{Calc}$ & Exp $^{*}$ \\
\hline $\mathrm{Ti}$ & 2.080 & 2.03 & 2.246 & - \\
\hline $\mathrm{V}$ & 2.034 & 1.994 & 2.188 & 2.14 \\
\hline $\mathrm{Cr}$ & 2.010 & 1.960 & 2.220 & 2.20 \\
\hline $\mathrm{Mn}$ & 2.086 & 1.991 & 2.236 & 2.20 \\
\hline $\mathrm{Fe}$ & 2.057 & 2.000 & 2.180 & 2.12 \\
\hline $\mathrm{Co}$ & 2.040 & 1.870 & 2.166 & 2.08 \\
\hline
\end{tabular}

*Persson I, Hydrated metal ions in aqueous solution: how regular are their structures?, Pure Appl. Chem., 82, 10, 1901-1917 (2010) doi:10.1351/PAC-CON-09-10-22

Table S2: Calculated redox potentials $\mathrm{M}(\mathrm{III}) / \mathrm{M}(\mathrm{II})$ in comparison with experimental data

\begin{tabular}{|c|c|c|c|}
\hline \multirow{2}{*}{$\mathrm{M}$} & \multicolumn{2}{|c|}{$\mathrm{E}^{\mathrm{o}}, \mathrm{V}$} & $\begin{array}{c}\text { Absolute/relative error, } \\
\mathrm{V} / \%\end{array}$ \\
\cline { 2 - 3 } & $\mathrm{Calc}$ & Exp* $^{*}$ & $0.036 / 9.8$ \\
\hline $\mathrm{Ti}$ & -0.404 & -0.368 & $0.019 / 7.6$ \\
\hline $\mathrm{V}$ & -0.236 & -0.255 & $0.027 / 6.7$ \\
\hline $\mathrm{Cr}$ & -0.434 & -0.407 & $0.142 / 9.4$ \\
\hline $\mathrm{Mn}$ & 1.367 & 1.509 & $0.015 / 1.9$ \\
\hline $\mathrm{Fe}$ & 0.786 & 0.771 & $0.076 / 4.2$ \\
\hline $\mathrm{Co}$ & 1.732 & 1.808 & \\
\hline
\end{tabular}

* Bard AJ, Parsons R, Jordan J (1985) Standard Potentials in Aqueous Solutions. Marcel Dekker, New York, p. 129.Google Scholar

P. Vanýsek, 2012/2013, p. "Electrochemical Series". Electrochemical Series. In: Haynes, W. M. Handbook of Chemistry and Physics: 93rd Edition. Chemical Rubber Company. p. 5-80. ISBN 9781439880494. 
TABLE S3: Atomic Cartesian coordinates (in $\AA$ ) for starting structure of complexes $\left[\mathrm{Fe}\left(\mathrm{H}_{2} \mathrm{O}\right)_{6} \cdot 12 \mathrm{H}_{2} \mathrm{O}\right]^{3+/ 2+}$

\begin{tabular}{cccc} 
Atom & $\mathrm{x}$ & $\mathrm{y}$ & $\mathrm{z}$ \\
$\mathrm{Fe}$ & -0.00080815 & -0.00329147 & -0.00084137 \\
$\mathrm{O}$ & -0.00498789 & -0.00292580 & 2.09056874 \\
$\mathrm{O}$ & 2.08592388 & 0.00004627 & -0.00069057 \\
$\mathrm{O}$ & 0.00368240 & 0.00117697 & -2.09201672 \\
$\mathrm{O}$ & -2.08698592 & -0.00300468 & -0.00082108 \\
$\mathrm{O}$ & 0.00176804 & 2.09445684 & 0.00333973 \\
$\mathrm{O}$ & -0.00190391 & -2.09973712 & -0.00536920 \\
$\mathrm{H}$ & 0.00226152 & 2.68275930 & 0.82760601 \\
$\mathrm{H}$ & 0.00367008 & 2.68478895 & -0.81840954 \\
$\mathrm{H}$ & -0.00534764 & -2.69562299 & 0.82527101 \\
$\mathrm{H}$ & -0.00068324 & -2.68640502 & -0.83151895 \\
$\mathrm{H}$ & 0.81596714 & -0.00038559 & 2.68108954 \\
$\mathrm{H}$ & -0.83078255 & -0.00219215 & 2.67951112 \\
$\mathrm{H}$ & 0.82872167 & 0.00131546 & -2.68126377 \\
$\mathrm{H}$ & -0.81761699 & -0.00084268 & -2.68148722 \\
$\mathrm{H}$ & 2.67495164 & 0.82331069 & -0.00201441 \\
$\mathrm{H}$ & 2.67539168 & -0.82212650 & -0.00497509 \\
$\mathrm{H}$ & -2.67456169 & 0.82054695 & 0.00372222 \\
$\mathrm{H}$ & -2.67567537 & -0.82800430 & -0.00029767 \\
$\mathrm{O}$ & 2.38260526 & 0.00986662 & 2.93248722 \\
$\mathrm{H}$ & 2.55164408 & 0.00578676 & 1.97636430 \\
$\mathrm{H}$ & 3.05227346 & -0.52533368 & 3.38449622 \\
$\mathrm{O}$ & 0.00554781 & -2.93539880 & -2.39682617 \\
$\mathrm{H}$ & 0.00473178 & -1.97948380 & -2.56422886 \\
$\mathrm{H}$ & -0.53017961 & -3.38142320 & -3.07035701 \\
$\mathrm{O}$ & -2.92555424 & 2.38688660 & 0.01815045 \\
$\mathrm{H}$ & -1.96901138 & 2.55449570 & 0.01389723 \\
$\mathrm{H}$ & -3.37523412 & 3.06198119 & -0.51180266 \\
$\mathrm{O}$ & -2.92866469 & -2.39048115 & 0.00473381 \\
$\mathrm{H}$ & -1.97298991 & -2.56181736 & 0.00061844 \\
$\mathrm{H}$ & -3.38085280 & -3.05634713 & -0.53501554 \\
$\mathrm{O}$ & -0.00135668 & 2.93225435 & 2.39322918 \\
$\mathrm{H}$ & -0.00173984 & 1.97652642 & 2.56064609 \\
$\mathrm{H}$ & 0.53806947 & 3.37796482 & 3.06407132 \\
$\mathrm{O}$ & -2.39289985 & -0.00410165 & 2.93031541 \\
$\mathrm{H}$ & -2.56433979 & -0.00270892 & 1.97527320 \\
$\mathrm{H}$ & -3.05633096 & 0.54204716 & 3.37954574 \\
$\mathrm{O}$ & -0.02389492 & -2.93040750 & 2.38135495 \\
$\mathrm{H}$ & -0.01705975 & -1.98107086 & 2.55256891 \\
$\mathrm{H}$ & 0.65158889 & -3.59173507 & 2.54434132 \\
$\mathrm{O}$ & 2.92824928 & -2.38738688 & -0.01856334 \\
$\mathrm{H}$ & 1.97140817 & -2.55565850 & -0.01503515 \\
& 3.38036509 & -3.06185452 & 0.50990244 \\
$\mathrm{H}$ & 2.92831097 & 2.38679259 & -0.00947303 \\
\hline
\end{tabular}



$\begin{array}{llll}\mathrm{O} & 0.01124280 & 2.93853133 & -2.38443260\end{array}$
H $\quad 0.00837286 \quad 1.98259332-2.55369243$
$\mathrm{H} \quad-0.52111296 \quad 3.38972970-3.05663162$
O $2.39122587-0.00383413-2.93201301$
H $\quad 2.56249640-0.00199774-1.97701483$
$\mathrm{H} \quad 3.05629013 \quad 0.54007253 \quad-3.38146823$
O $\quad-2.38548255-0.01080638-2.93349048$
$\mathrm{H} \quad-2.55291520-0.00770746-1.97701551$
$\mathrm{H} \quad-3.05732699 \quad 0.52396543 \quad-3.38249235$

TABLE S4: Atomic Cartesian coordinates (in $\AA$ ) for complexes $\left[\mathrm{Fe}\left(\mathrm{H}_{2} \mathrm{O}\right)_{6} \cdot 12 \mathrm{H}_{2} \mathrm{O}\right]^{3+/ 2+}$ optimized at the PBE/TZVP level

\section{$\left[\mathrm{Fe}\left(\mathrm{H}_{2} \mathrm{O}\right)_{6} \cdot 12 \mathrm{H}_{2} \mathrm{O}\right]^{3+}$}

$\begin{array}{cccc}\text { Atom } & \mathrm{x} & \mathrm{y} & \mathrm{z} \\ \mathrm{Fe} & -0.028532 & 0.019120 & 0.110164 \\ \mathrm{O} & -1.726834 & -0.143504 & 1.228614 \\ \mathrm{O} & 0.812364 & 1.413501 & 1.330564 \\ \mathrm{O} & 1.647329 & 0.109644 & -1.116478 \\ \mathrm{O} & -0.924417 & -1.219536 & -1.333462 \\ \mathrm{O} & -0.894961 & 1.639453 & -0.927821 \\ \mathrm{O} & 0.786750 & -1.490304 & 1.114853 \\ \mathrm{H} & -1.880375 & 1.820011 & -0.940126 \\ \mathrm{H} & -0.431276 & 2.274557 & -1.532372 \\ \mathrm{H} & 0.410787 & -1.890931 & 1.978173 \\ \mathrm{H} & 1.745840 & -1.838006 & 0.939263 \\ \mathrm{H} & -2.192643 & 0.593701 & 1.693770 \\ \mathrm{H} & -2.383774 & -0.945254 & 1.149900 \\ \mathrm{H} & 2.493289 & 0.528237 & -0.748148 \\ \mathrm{H} & 1.917379 & -0.696285 & -1.652151 \\ \mathrm{H} & 1.068365 & 2.298415 & 0.945425 \\ \mathrm{H} & 1.608430 & 1.113771 & 1.929238 \\ \mathrm{H} & -1.886511 & -1.080924 & -1.587179 \\ \mathrm{H} & -0.650308 & -2.130145 & -1.612742 \\ \mathrm{O} & -3.621846 & 1.859436 & 2.016576 \\ \mathrm{O} & 3.135876 & -2.284084 & 0.473493 \\ \mathrm{O} & -3.586636 & -0.804202 & -1.676849 \\ \mathrm{O} & 0.042391 & -3.616632 & -2.499212 \\ \mathrm{O} & -3.602253 & 1.865720 & -0.773914 \\ \mathrm{O} & -3.343460 & -2.059018 & 0.801252 \\ \mathrm{O} & -0.166939 & -2.532163 & 3.303475 \\ \mathrm{O} & 2.917400 & 0.647858 & 2.551530 \\ \mathrm{O} & 1.996816 & 3.584826 & 0.078483 \\ \mathrm{O} & 0.502230 & 3.671436 & -2.318761 \\ \mathrm{O} & 3.744359 & 1.425256 & -0.081681 \\ \mathrm{O} & 2.467979 & -2.230414 & -2.246410 \\ \mathrm{H} & -3.768769 & -1.854152 & -0.063704 \\ \mathrm{H} & -3.992713 & -2.502469 & 1.374296 \\ \mathrm{H} & -4.411647 & 1.534032 & 2.491021 \\ \mathrm{H} & -3.387565 & 2.688854 & 2.475979\end{array}$




$\begin{array}{crrr}\mathrm{H} & -4.151357 & 2.536433 & -1.221747 \\ \mathrm{H} & -3.781879 & 1.957075 & 0.200999 \\ \mathrm{H} & 0.022355 & 4.499730 & -2.514606 \\ \mathrm{H} & 0.980570 & 3.464143 & -3.144689 \\ \mathrm{H} & -4.121804 & -1.020874 & -2.462393 \\ \mathrm{H} & -3.768593 & 0.146430 & -1.456423 \\ \mathrm{H} & -0.283233 & -3.762376 & -3.408855 \\ \mathrm{H} & -0.009872 & -4.494483 & -2.074067 \\ \mathrm{H} & 3.092192 & -2.297536 & -2.992761 \\ \mathrm{H} & 1.704580 & -2.828183 & -2.457690 \\ \mathrm{H} & 4.630101 & 1.457816 & -0.487857 \\ \mathrm{H} & 3.387850 & 2.351101 & -0.079840 \\ \mathrm{H} & 2.203232 & 4.442865 & 0.494042 \\ \mathrm{H} & 1.563361 & 3.786500 & -0.790002 \\ \mathrm{H} & 3.286805 & 0.983278 & 3.388137 \\ \mathrm{H} & 3.592423 & 0.787170 & 1.849743 \\ \mathrm{H} & -0.300258 & -3.491353 & 3.408868 \\ \mathrm{H} & 0.088048 & -2.200587 & 4.183070 \\ \mathrm{H} & 3.713578 & -2.904886 & 0.950997 \\ \mathrm{H} & 3.146038 & -2.520986 & -0.483858\end{array}$

\section{$\left[\mathrm{Fe}\left(\mathrm{H}_{2} \mathrm{O}\right)_{6} \cdot \mathbf{1 2} \mathrm{H}_{2} \mathrm{O}\right]^{2+}$}

$\begin{array}{crcc}\text { Atom } & \mathrm{x} & \mathrm{y} & \mathrm{z} \\ \mathrm{Fe} & -0.000197 & 0.000417 & -0.000002 \\ \mathrm{O} & -1.443739 & -0.958007 & 1.506586 \\ \mathrm{O} & -1.364122 & -0.512607 & -1.619777 \\ \mathrm{O} & 1.443855 & 0.959000 & -1.506450 \\ \mathrm{O} & 1.364486 & 0.513047 & 1.619125 \\ \mathrm{O} & 0.871656 & -1.907220 & -0.223374 \\ \mathrm{O} & -0.872187 & 1.907935 & 0.222513 \\ \mathrm{H} & 0.396120 & -2.689397 & 0.162977 \\ \mathrm{H} & 1.794893 & -2.178315 & -0.464834 \\ \mathrm{H} & -1.795278 & 2.178971 & 0.464593 \\ \mathrm{H} & -0.396452 & 2.690066 & -0.163604 \\ \mathrm{H} & -2.395987 & -0.677412 & 1.446685 \\ \mathrm{H} & -1.116894 & -0.741663 & 2.446521 \\ \mathrm{H} & 1.117288 & 0.740705 & -2.446057 \\ \mathrm{H} & 2.395789 & 0.677468 & -1.446031 \\ \mathrm{H} & -1.660244 & -1.491545 & -1.620078 \\ \mathrm{H} & -2.215511 & 0.004626 & -1.612571 \\ \mathrm{H} & 2.216465 & -0.003178 & 1.611053 \\ \mathrm{H} & 1.659325 & 1.492398 & 1.621042 \\ \mathrm{O} & -4.106263 & -0.198575 & 1.166871 \\ \mathrm{O} & 0.994126 & 3.554380 & -0.855749 \\ \mathrm{O} & 3.867966 & -0.691491 & 1.452052 \\ \mathrm{O} & 2.286987 & 2.946267 & 1.479096 \\ \mathrm{O} & -0.993923 & -3.554194 & 0.856095 \\ \mathrm{O} & -0.204698 & -0.455215 & 3.726659 \\ \mathrm{O} & -3.543097 & 2.454099 & 0.657908\end{array}$




$\begin{array}{crcc}\mathrm{O} & -3.866461 & 0.693432 & -1.452943 \\ \mathrm{O} & -2.289776 & -2.944648 & -1.475970 \\ \mathrm{O} & 3.542290 & -2.454367 & -0.657515 \\ \mathrm{O} & 0.207743 & 0.449213 & -3.726762 \\ \mathrm{O} & 4.105903 & 0.197904 & -1.168417 \\ \mathrm{H} & -0.428257 & -0.007953 & 4.559714 \\ \mathrm{H} & 0.507868 & 0.059358 & 3.280538 \\ \mathrm{H} & -4.839837 & -0.692439 & 1.574489 \\ \mathrm{H} & -4.255455 & -0.208681 & 0.188113 \\ \mathrm{H} & -1.002552 & -4.278509 & 1.505177 \\ \mathrm{H} & -1.286658 & -2.716953 & 1.315474 \\ \mathrm{H} & 3.938791 & -3.278450 & -0.990845 \\ \mathrm{H} & 3.912075 & -1.707051 & -1.188935 \\ \mathrm{H} & 4.469293 & -0.748397 & 2.215662 \\ \mathrm{H} & 3.863735 & -1.578801 & 1.015773 \\ \mathrm{H} & 2.409725 & 3.595137 & 2.191548 \\ \mathrm{H} & 1.910448 & 3.424259 & 0.696890 \\ \mathrm{H} & 4.839027 & 0.690646 & -1.578149 \\ \mathrm{H} & 4.257299 & 0.208680 & -0.190046 \\ \mathrm{H} & 0.432019 & 0.002155 & -4.559646 \\ \mathrm{H} & -0.505973 & -0.064482 & -3.281563 \\ \mathrm{H} & -2.417443 & -3.593236 & -2.187789 \\ \mathrm{H} & -1.912538 & -3.423577 & -0.694649 \\ \mathrm{H} & -4.467885 & 0.751792 & -2.216357 \\ \mathrm{H} & -3.862274 & 1.580011 & -1.015062 \\ \mathrm{H} & -3.939996 & 3.278298 & 0.990520 \\ \mathrm{H} & -3.913152 & 1.706910 & 1.189224 \\ \mathrm{H} & 1.003624 & 4.278905 & -1.504569 \\ \mathrm{H} & 1.287757 & 2.717186 & -1.314745\end{array}$

TABLE S5: Atomic Cartesian coordinates (in $\AA$ ) for inclusion compounds $\left[\mathrm{Fe}\left(\mathrm{H}_{2} \mathrm{O}\right)_{6}\right]^{3+/ 2+} @ \mathrm{CB}[6]$ optimized at the PBE/TZVP level

\begin{tabular}{|c|c|c|c|}
\hline \\
\hline \multicolumn{4}{|c|}{ Atom $\mathrm{x}$} \\
\hline $\mathrm{N}$ & -3.933409 & 1.987151 & -1.10 \\
\hline $\mathrm{C}$ & -4.863806 & 7043 & 16479 \\
\hline $\mathrm{C}$ & -5.191127 & 3582 & \\
\hline $\mathrm{N}$ & 0947 & 1095 & 968 \\
\hline $\mathrm{C}$ & -3.584846 & & 583 \\
\hline $\mathrm{N}$ & -4.715485 & 1944 & 313 \\
\hline $\mathrm{C}$ & -4.1 & & \\
\hline $\mathrm{N}$ & -4.2 & & 563 \\
\hline C & -3.642239 & 0946 & 5209 \\
\hline N & -2.3 & 772 & 1.0 \\
\hline $\mathrm{C}$ & -2.341204 & 4.641909 & -0.015730 \\
\hline $\mathrm{C}$ & -0.840881 & 5.130410 & -0.015952 \\
\hline $\mathrm{N}$ & -0.246011 & 4.399844 & 1.100757 \\
\hline $\mathrm{C}$ & -1.134462 & 3.483855 & 1.611774 \\
\hline
\end{tabular}




\begin{tabular}{|c|c|c|c|}
\hline & -2.488864 & 4.007817 & \\
\hline & -1.306374 & 4.009936 & -2.053304 \\
\hline & -0.348395 & 702334 & -1.310882 \\
\hline & 1.011768 & & \\
\hline & 2.225733 & 4.157520 & 1.311404 \\
\hline & 2.849533 & & 0.016411 \\
\hline & 4.02 & & \\
\hline & 3.899278 & 2.653317 & 1361 \\
\hline & 2.819890 & & 3471 \\
\hline & 2.00 & & \\
\hline & 2.4 & & \\
\hline & 3.68 & 021 & 095 \\
\hline & 4.6 & & \\
\hline & 4.4 & & \\
\hline & 5.1 & -0.2 & \\
\hline & 4.8 & -1.8 & \\
\hline & 3.9 & & \\
\hline & & & \\
\hline & 4.7 & & \\
\hline & 4.12 & -0.8 & \\
\hline & 4.2 & & \\
\hline & 3.6 & -3.2 & \\
\hline & 2.4 & & \\
\hline & 2.3 & -4.6 & \\
\hline & 0.8 & & \\
\hline & & & \\
\hline & 1.3 & -4.0 & \\
\hline & 2.3 & -3.7 & \\
\hline & 1.1 & -3.4 & \\
\hline & 0.2 & -4.3 & \\
\hline & -1.02 & -4.7 & \\
\hline & -2.0 & -3.9 & 494 \\
\hline & -2.8 & -4.3 & -0. \\
\hline & -4.02 & -3.2 & 5894 \\
\hline & -3.68 & -2.4 & \\
\hline & -2.4 & -2.7 & \\
\hline $\mathrm{N}$ & -2.22 & -4.1 & -1.2 \\
\hline & -2.81 & -3.1 & 3778 \\
\hline & -3.8 & & \\
\hline & -4.66 & -1.5 & 4977 \\
\hline $\mathrm{C}$ & -1.01 & -4.79 & 5264 \\
\hline $\mathrm{O}$ & -2.46 & -2.73 & 7659 \\
\hline $\mathrm{C}$ & -4.66 & -1.5 & \\
\hline $\mathrm{O}$ & -1.797925 & -1.997133 & 2.395342 \\
\hline $\mathrm{O}$ & 1.140537 & -3.50 & 3.147394 \\
\hline $\mathrm{O}$ & $0.8 ?$ & & \\
\hline $\mathrm{C}$ & 3.642964 & -3.282033 & -1.764758 \\
\hline $\mathrm{O}$ & 2.628663 & -0.557006 & 2.395144 \\
\hline $\mathrm{O}$ & 3.602413 & -0.763256 & -3.147467 \\
\hline $\mathrm{C}$ & 4.660903 & 1.522840 & -1.764947 \\
\hline
\end{tabular}




\begin{tabular}{|c|c|c|c|}
\hline & .462535 & 737873 & \\
\hline & 796689 & .997820 & \\
\hline & 1.020742 & 795359 & -1.76 \\
\hline 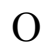 & -0.831606 & & \\
\hline & -1.141058 & 3.501704 & -3.147380 \\
\hline & -3.649787 & 3.275475 & 4621 \\
\hline J & -3.603467 & & \\
\hline ) & -2.628363 & 0.557259 & \\
\hline & 3.074869 & & -0.1 \\
\hline & 0.725023 & & \\
\hline $\mathrm{H}$ & 6.258 & -0.0 & \\
\hline & 5.746773 & -2.4 & \\
\hline & 85 & & \\
\hline & 5.02 & & \\
\hline $\mathrm{I}$ & -3.074073 & & \\
\hline 1 & -0.724777 & 6.2 & \\
\hline & -6.258 & 0.0 & \\
\hline $\mathrm{H}$ & -5.7 & & \\
\hline I & -3.182 & -5.3 & \\
\hline & -5.021 & -3.7 & \\
\hline I & -5.74 & -1.7 & \\
\hline $\mathrm{H}$ & -4.4 & & \\
\hline H & -1.09 & & \\
\hline & -0.92 & -4.5 & -2 \\
\hline $\mathrm{H}$ & 4.3 & & \\
\hline H & 3.4 & & \\
\hline & 5.64 & & -1 . \\
\hline & 4.41 & & \\
\hline $\mathrm{H}$ & 1.38 & & \\
\hline $\mathrm{H}$ & 1.00 & & \\
\hline $\mathrm{H}$ & -4.55 & 3.8 & -1. \\
\hline & -3.4 & 3.0 & \\
\hline $\mathrm{H}$ & -5.6 & -1.9 & \\
\hline $\mathrm{H}$ & -4.41 & -1.4 & 2. \\
\hline H & -4.35 & 4.1 & \\
\hline 1 & -3.4 & 3.1 & \\
\hline $\mathrm{H}$ & 1.094 & & \\
\hline $\mathrm{H}$ & 0.926161 & & \\
\hline & 5.72 & & \\
\hline $\mathrm{H}$ & 4.435 & & \\
\hline $\mathrm{H}$ & 4.55 & -3.8 & \\
\hline 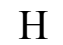 & 3.48 & -3.0 & \\
\hline $\mathrm{H}$ & -1.38 & -5.8 & \\
\hline $\mathrm{H}$ & -1.002318 & -4.540479 & 2.833927 \\
\hline $\mathrm{Fe}$ & -0.000090 & 0.000068 & -0.00001 \\
\hline $\mathrm{O}$ & & & \\
\hline $\mathrm{O}$ & 0.489012 & -1.503561 & 1.140853 \\
\hline $\mathrm{O}$ & -1.057517 & -1.175205 & -1.14103 \\
\hline $\mathrm{O}$ & -0.489367 & 1.503801 & -1.14066 \\
\hline $\mathrm{H}$ & 0.260402 & 1.813662 & -1.745711 \\
\hline
\end{tabular}




$\begin{array}{rrrr}\mathrm{H} & -1.277974 & 1.313121 & -1.745577 \\ \mathrm{H} & -0.260768 & -1.813294 & 1.745901 \\ \mathrm{H} & 1.277713 & -1.313043 & 1.745654 \\ \mathrm{H} & -0.497884 & -1.762747 & -1.745839 \\ \mathrm{H} & -1.700724 & -0.680686 & -1.746102 \\ \mathrm{H} & 1.700992 & 0.680700 & 1.745720 \\ \mathrm{H} & 0.498092 & 1.762689 & 1.745950 \\ \mathrm{O} & -1.547015 & 0.328068 & 1.140679 \\ \mathrm{O} & 1.546766 & -0.327859 & -1.140783 \\ \mathrm{H} & 1.440277 & -1.132258 & -1.745729 \\ \mathrm{H} & 1.775935 & 0.450416 & -1.745728 \\ \mathrm{H} & -1.440201 & 1.132283 & 1.745772 \\ \mathrm{H} & -1.776476 & -0.450227 & 1.745532\end{array}$

\section{$\left[\mathrm{Fe}\left(\mathrm{H}_{2} \mathrm{O}\right)_{6}\right]^{2+} @ \mathrm{CB}[6]$}

$\begin{array}{cccc}\text { Atom } & \mathrm{x} & \mathrm{y} & \mathrm{Z} \\ \mathrm{N} & -1.171054 & -4.233734 & 1.117639 \\ \mathrm{C} & -1.899985 & -4.808977 & -0.005499 \\ \mathrm{C} & -3.263245 & -4.017998 & -0.005902 \\ \mathrm{~N} & -3.126233 & -3.099459 & 1.116816 \\ \mathrm{C} & -1.875240 & -3.195337 & 1.697074 \\ \mathrm{~N} & -3.286458 & -3.386201 & -1.309455 \\ \mathrm{C} & -2.161927 & -3.691802 & -2.080398 \\ \mathrm{~N} & -1.339569 & -4.516575 & -1.309214 \\ \mathrm{C} & -0.021636 & -4.899951 & -1.764790 \\ \mathrm{~N} & 1.126792 & -4.245133 & -1.116732 \\ \mathrm{C} & 1.849830 & -4.828034 & 0.006259 \\ \mathrm{C} & 3.221208 & -4.051257 & 0.006698 \\ \mathrm{~N} & 3.093817 & -3.131315 & -1.116029 \\ \mathrm{C} & 1.842176 & -3.214770 & -1.696885 \\ \mathrm{~N} & 1.292562 & -4.529873 & 1.309995 \\ \mathrm{C} & 2.123204 & -3.713395 & 2.081060 \\ \mathrm{~N} & 3.251025 & -3.419820 & 1.310272 \\ \mathrm{C} & 4.245584 & -2.479756 & -1.762081 \\ \mathrm{~N} & 4.588575 & -1.149791 & -1.308281 \\ \mathrm{C} & 5.123596 & -0.814686 & -0.004068 \\ \mathrm{C} & 5.131692 & 0.761737 & -0.004202 \\ \mathrm{~N} & 4.598263 & 1.101996 & -1.307635 \\ \mathrm{C} & 4.291841 & -0.022322 & -2.078396 \\ \mathrm{~N} & 4.256654 & -1.151871 & 1.117947 \\ \mathrm{C} & 3.716748 & -0.019012 & 1.695834 \\ \mathrm{~N} & 4.269745 & 1.107889 & 1.118834 \\ \mathrm{C} & 4.271586 & 2.435714 & -1.762436 \\ \mathrm{~N} & 3.127151 & 3.101109 & -1.117707 \\ \mathrm{C} & 3.263048 & 4.018381 & 0.006113 \\ \mathrm{C} & 1.899638 & 4.809113 & 0.005282 \\ \mathrm{~N} & 1.170737 & 4.232995 & -1.117461 \\ \mathrm{C} & 1.875846 & 3.195323 & -1.697235 \\ \mathrm{~N} & 3.284849 & 3.384887 & 1.308919\end{array}$




\begin{tabular}{|c|c|c|c|}
\hline & 2.161274 & 3.692262 & \\
\hline & & & 1309172 \\
\hline & 0.029004 & & \\
\hline & -1.292536 & 4.529088 & -1.309755 \\
\hline & .850240 & 4.828614 & \\
\hline & -3.221508 & & \\
\hline & -3.251387 & 3.419734 & \\
\hline & -2.123431 & 3.712858 & -2.080766 \\
\hline & -1.127787 & & \\
\hline & -1.841 & & \\
\hline & -3.093698 & & \\
\hline & -4.247106 & 4742 & \\
\hline & -4.257 & & \\
\hline & -5.12 & & \\
\hline & -5.13 & & \\
\hline & -4.26 & -1.1 & -1.11 \\
\hline & -3.7 & & \\
\hline & -4.5 & & \\
\hline & -4.29 & & \\
\hline & -4.5 & -1.1 & \\
\hline & $-4.2^{\prime}$ & -2.4 & \\
\hline y & -4.2 & & \\
\hline O & $-3.8^{\prime}$ & 0.0 & \\
\hline & $-4.2^{\prime}$ & -2.4 & -1.7 \\
\hline 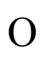 & -2.8 & & -2.5 \\
\hline O & -1.91 & & -3.2 \\
\hline 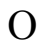 & -1.41 & 2.4 & \\
\hline & 0.02 & 4.9 & \\
\hline D & 1.4 & & -2.5 \\
\hline D & 1.9 & & 3.2 \\
\hline & $4.2^{7}$ & 2.4 & \\
\hline D & $3.8^{\prime}$ & -0.0 & -3.2 \\
\hline $\mathrm{O}$ & 2.85 & -0.0 & \\
\hline & 4.24 & -2.4 & \\
\hline O & 1.4 & -2.4 & -2.5 \\
\hline O & $1.91^{\circ}$ & -3.3 & \\
\hline $\mathrm{C}$ & -0.02 & -4.9 & \\
\hline $\mathrm{O}$ & -1.9 & -3.3 & -3.22 \\
\hline $\mathrm{O}$ & -1.4 & -2.4 & \\
\hline $\mathrm{H}$ & -1.960 & & \\
\hline $\mathrm{H}$ & -4.098 & 4.7 & \\
\hline & 4.14 & 4.6 & \\
\hline $\mathrm{H}$ & 2.020796 & & -0.13 \\
\hline $\mathrm{H}$ & 6.12 & -1.260329 & \\
\hline $\mathrm{H}$ & 6.13 & & \\
\hline $\mathrm{H}$ & 1.959 & -5.9 & -0.1 \\
\hline $\mathrm{H}$ & 4.097703 & -4.705022 & -0.137062 \\
\hline $\mathrm{H}$ & -4.146493 & -4.662615 & 0.137807 \\
\hline $\mathrm{H}$ & -2.021329 & -5.895590 & \\
\hline & -6.121683 & 1.260447 & -0.14046 \\
\hline
\end{tabular}




$\begin{array}{rrrr}\mathrm{H} & -6.134664 & -1.197055 & -0.140073 \\ \mathrm{H} & -5.138251 & -3.101701 & 1.628143 \\ \mathrm{H} & -4.050297 & -2.338016 & 2.835324 \\ \mathrm{H} & -5.105616 & 3.154886 & 1.627681 \\ \mathrm{H} & -4.025562 & 2.380105 & 2.834880 \\ \mathrm{H} & -0.119156 & 5.986816 & 1.632917 \\ \mathrm{H} & -0.004505 & 4.659556 & 2.836723 \\ \mathrm{H} & 5.284368 & 2.845326 & 1.629276 \\ \mathrm{H} & 4.076954 & 2.290653 & 2.836847 \\ \mathrm{H} & 5.255242 & -2.898283 & 1.630295 \\ \mathrm{H} & 4.052770 & -2.331316 & 2.837154 \\ \mathrm{H} & -0.181284 & -5.983673 & 1.634271 \\ \mathrm{H} & -0.052899 & -4.656986 & 2.837394 \\ \mathrm{H} & -5.285383 & -2.844602 & -1.629571 \\ \mathrm{H} & -4.077343 & -2.290215 & -2.836693 \\ \mathrm{H} & 0.119607 & -5.985101 & -1.633728 \\ \mathrm{H} & 0.004531 & -4.656946 & -2.836603 \\ \mathrm{H} & 5.105781 & -3.155264 & -1.627252 \\ \mathrm{H} & 4.026148 & -2.380260 & -2.834680 \\ \mathrm{H} & 5.139586 & 3.101091 & -1.627198 \\ \mathrm{H} & 4.051838 & 2.338355 & -2.835163 \\ \mathrm{H} & 0.181149 & 5.982870 & -1.634837 \\ \mathrm{H} & 0.052617 & 4.655704 & -2.837402 \\ \mathrm{H} & -5.255616 & 2.898207 & -1.630068 \\ \mathrm{H} & -4.053165 & 2.331355 & -2.836996 \\ \mathrm{Fe} & 0.000076 & -0.000527 & -0.000254 \\ \mathrm{O} & 1.670477 & -0.009670 & -1.350776 \\ \mathrm{O} & -0.816022 & 1.434409 & -1.382145 \\ \mathrm{O} & -1.670208 & 0.008902 & 1.350404 \\ \mathrm{O} & 0.816405 & -1.434760 & 1.382133 \\ \mathrm{H} & 1.531585 & -1.092569 & 1.978397 \\ \mathrm{H} & 0.152108 & -1.842443 & 1.995844 \\ \mathrm{H} & -1.531148 & 1.092600 & -1.978648 \\ \mathrm{H} & -0.151288 & 1.841869 & -1.995533 \\ \mathrm{H} & -1.716942 & 0.792642 & 1.955280 \\ \mathrm{H} & -1.725337 & -0.774024 & 1.955646 \\ \mathrm{H} & 1.725981 & 0.773785 & -1.955323 \\ \mathrm{H} & 1.717282 & -0.792857 & -1.956344 \\ \mathrm{O} & -0.830909 & -1.426685 & -1.382767 \\ \mathrm{O} & 0.831228 & 1.426560 & 1.381453 \\ \mathrm{H} & 0.171189 & 1.841331 & 1.994993 \\ \mathrm{H} & 1.542777 & 1.077261 & 1.977906 \\ \mathrm{H} & -0.171056 & -1.841088 & -1.996679 \\ \mathrm{H} & -1.542851 & -1.077372 & -1.978783\end{array}$

TABLE S6: Atomic Cartesian coordinates (in $\AA$ ) for inclusion compounds $\left[\mathrm{Fe}\left(\mathrm{H}_{2} \mathrm{O}\right)_{6} \cdot 4 \mathrm{H}_{2} \mathrm{O}\right]^{3+/ 2+}$ @ CB[7] optimized at the PBE/TZVP level

$\left[\mathrm{Fe}\left(\mathrm{H}_{2} \mathrm{O}\right)_{6} \cdot 4 \mathrm{H}_{2} \mathrm{O}\right]^{3+} @ \mathrm{CB}[7]$

Atom $\mathrm{x} \quad \mathrm{y} \quad \mathrm{z}$ 


\begin{tabular}{lrrr}
$\mathrm{C}$ & -1.855966 & -4.527733 & 1.752624 \\
$\mathrm{~N}$ & -3.005381 & -4.454445 & 0.986123 \\
$\mathrm{C}$ & -2.852710 & -5.116048 & -0.304890 \\
$\mathrm{C}$ & -1.342191 & -5.552894 & -0.315132 \\
$\mathrm{~N}$ & -0.874078 & -5.165083 & 1.009683 \\
$\mathrm{~N}$ & -0.769719 & -4.787456 & -1.414317 \\
$\mathrm{C}$ & -1.739928 & -4.035800 & -2.096582 \\
$\mathrm{~N}$ & -2.956724 & -4.245455 & -1.457133 \\
$\mathrm{C}$ & -4.190304 & -3.724232 & -2.003923 \\
$\mathrm{~N}$ & -4.694113 & -2.519018 & -1.362529 \\
$\mathrm{C}$ & -5.496870 & -2.513503 & -0.145968 \\
$\mathrm{C}$ & -5.960118 & -1.017855 & -0.035225 \\
$\mathrm{~N}$ & -5.409013 & -0.397226 & -1.233091 \\
$\mathrm{C}$ & -4.659713 & -1.288724 & -1.979992 \\
$\mathrm{~N}$ & -4.757763 & -2.735858 & 1.080762 \\
$\mathrm{C}$ & -4.725988 & -1.607292 & 1.899714 \\
$\mathrm{~N}$ & -5.362938 & -0.574005 & 1.206228 \\
$\mathrm{C}$ & -5.839869 & 0.891628 & -1.749643 \\
$\mathrm{~N}$ & -5.160981 & 2.048084 & -1.191580 \\
$\mathrm{C}$ & -5.457033 & 2.627593 & 0.110598 \\
$\mathrm{C}$ & -4.574287 & 3.930616 & 0.130800 \\
$\mathrm{~N}$ & -3.945339 & 3.932826 & -1.180430 \\
$\mathrm{C}$ & -4.260159 & 2.806898 & -1.918504 \\
$\mathrm{~N}$ & -4.947753 & 1.851269 & 1.228088 \\
$\mathrm{C}$ & -3.830754 & 2.435382 & 1.794949 \\
$\mathrm{~N}$ & -3.649335 & 3.684105 & 1.226620 \\
$\mathrm{C}$ & -3.142624 & 5.019735 & -1.728062 \\
$\mathrm{~N}$ & -1.769322 & 5.096056 & -1.267353 \\
$\mathrm{C}$ & -1.332156 & 5.720971 & -0.041949 \\
$\mathrm{C}$ & 0.224783 & 5.758032 & -0.188540 \\
$\mathrm{~N}$ & 0.469612 & 4.789308 & -1.260365 \\
$\mathrm{C}$ & -0.720140 & 4.486327 & -1.957666 \\
$\mathrm{~N}$ & 3.514352 & 3.364537 & 1.801970 \\
$\mathrm{C}$ & 2.999080 & 4.410432 & 1.046163 \\
$\mathrm{O}$ & -2.072383 & 5.390438 & 1.584777 \\
$\mathrm{C}$ & -0.306173 & 5.317947 & 1.109861 \\
$\mathrm{~N}$ & -1.508145 & 4.668152 & 1.812003 \\
$\mathrm{C}$ & -5.632732 & 0.704917 & 1.153418 \\
$\mathrm{C}$ & -2.781994 & 4.685048 & 1.822412 \\
$\mathrm{O}$ & -3.826355 & 2.534774 & -3.039459 \\
$\mathrm{O}$ & -3.107428 & 1.932689 & 2.660385 \\
$\mathrm{C}$ & 1.734910 & 4.755509 & -1.987744 \\
$\mathrm{~N}$ & 2.776213 & 3.974178 & -1.349393 \\
$\mathrm{C}$ & 3.605826 & 4.494026 & -0.267457 \\
$\mathrm{~N}$ & 3.478762 & 3.463395 & -0.196431 \\
$\mathrm{~N}$ & 2.700965 & -1.410141 \\
$\mathrm{~N}$ & & -2.970291 \\
\hline
\end{tabular}




\begin{tabular}{|c|c|c|c|}
\hline & -0.152995 & 962050 & \\
\hline & 5.621217 & 767564 & \\
\hline & & & \\
\hline & 6.086007 & & \\
\hline & & .553991 & \\
\hline & 5.21 & 5944 & \\
\hline & & -0.635366 & \\
\hline & 6740 & -1.809172 & \\
\hline & 4.85 & 597 & \\
\hline & & & \\
\hline & 5.42 & 2711 & \\
\hline & 2.96 & 9917 & \\
\hline & $3.1^{\circ}$ & 536 & \\
\hline & & & \\
\hline & $3.6^{\prime}$ & 9504 & \\
\hline & 3.6 & -4.4 & \\
\hline & & & \\
\hline & & -4. & \\
\hline & 2.4 & -3.7 & \\
\hline & & -4.6 & \\
\hline & & & \\
\hline & & & \\
\hline & 4.8 & -3.1 & \\
\hline & 4.6 & -0. & -3.2 \\
\hline & & -0. & \\
\hline & & 082 & \\
\hline & 0.4 & -5.4 & \\
\hline 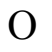 & & & \\
\hline D & 2.2 & -3. & \\
\hline & -4.2 & -4. & \\
\hline O & -4.0 & -1 . & \\
\hline ) & -4.2 & -1. & \\
\hline $\mathrm{O}$ & -1.5 & 8116 & \\
\hline D & -1.7 & 8754 & \\
\hline & -5.1 & & \\
\hline $\mathrm{H}$ & -6.5 & 2.8 & \\
\hline 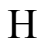 & 0.62 & 753 & -0.4 \\
\hline & -1.79 & 2343 & \\
\hline & $5.7^{\prime}$ & 053 & -0.1 \\
\hline $\mathrm{H}$ & 3.93 & 6025 & $-0 .{ }^{2}$ \\
\hline & 6.83 & 5807 & -0.0 \\
\hline & & & \\
\hline $\mathrm{H}$ & 2.230004 & -6.242642 & -0.22 \\
\hline $\mathrm{H}$ & 4.45 & -5.232255 & -0.2 \\
\hline $\mathrm{H}$ & -7.0 & 93945 & -0 . \\
\hline $\mathrm{H}$ & -6.329431 & -3.231574 & -0.23678 \\
\hline $\mathrm{H}$ & 0.696469 & -6.482421 & \\
\hline $\mathrm{H}$ & 0.422915 & -5.159441 & \\
\hline & -6.715225 & & \\
\hline & -5.305757 & 0.622879 & 2.86843 \\
\hline
\end{tabular}




\begin{tabular}{rrrr}
$\mathrm{H}$ & -3.332685 & 5.640509 & 1.857393 \\
$\mathrm{H}$ & -2.551360 & 4.356041 & 2.844463 \\
$\mathrm{H}$ & 2.451748 & 6.399903 & 1.361310 \\
$\mathrm{H}$ & 2.046810 & 5.240988 & 2.672487 \\
$\mathrm{H}$ & 6.453586 & 2.148056 & 1.492041 \\
$\mathrm{H}$ & 5.205863 & 1.714075 & 2.702064 \\
$\mathrm{H}$ & 5.684110 & -3.782843 & 1.477122 \\
$\mathrm{H}$ & 4.621767 & -3.007665 & 2.703111 \\
$\mathrm{H}$ & -4.966199 & -4.504859 & -1.946423 \\
$\mathrm{H}$ & -3.998708 & -3.470931 & -3.055846 \\
$\mathrm{H}$ & 5.718952 & -3.785888 & -1.740999 \\
$\mathrm{H}$ & 4.754471 & -2.945650 & -3.004023 \\
$\mathrm{H}$ & 6.618853 & 2.194804 & -1.731850 \\
$\mathrm{H}$ & 5.463619 & 1.670554 & -3.004084 \\
$\mathrm{H}$ & 2.095473 & 5.790162 & -2.138186 \\
$\mathrm{H}$ & 1.531170 & 4.292758 & -2.964431 \\
$\mathrm{H}$ & -3.639667 & 5.975891 & -1.503103 \\
$\mathrm{H}$ & -3.114702 & 4.867885 & -2.815733 \\
$\mathrm{H}$ & -6.922636 & 0.994445 & -1.569440 \\
$\mathrm{H}$ & -5.652143 & 0.900786 & -2.832124 \\
$\mathrm{H}$ & 0.399574 & -4.756577 & -3.123764 \\
$\mathrm{H}$ & 0.509580 & -6.271519 & -2.155385 \\
$\mathrm{H}$ & -5.045958 & -4.794605 & 1.293572 \\
$\mathrm{H}$ & -4.168627 & -3.970308 & 2.626095 \\
$\mathrm{H}$ & -1.197137 & -6.634388 & -0.476035 \\
$\mathrm{H}$ & -3.558211 & -5.959944 & -0.387692 \\
$\mathrm{Fe}$ & 0.028647 & -0.093979 & 0.646960 \\
$\mathrm{O}$ & 0.418928 & 1.946891 & 0.989988 \\
$\mathrm{O}$ & 0.750185 & -0.438499 & 2.469381 \\
$\mathrm{O}$ & -0.184972 & -2.168780 & 0.259483 \\
$\mathrm{O}$ & -0.474949 & 0.275595 & -1.193540 \\
$\mathrm{H}$ & 3.158632 & 0.189218 & 3.870396 \\
$\mathrm{O}$ & 2.057056 & -0.327438 & -0.068517 \\
$\mathrm{H}$ & -1.347744 & -1.619597 & 3.782795 \\
$\mathrm{O}$ & -1.857747 & -0.143076 & 1.503782 \\
$\mathrm{H}$ & 2.698356 & 0.306827 & 0.303338 \\
$\mathrm{H}$ & 2.125111 & -0.281900 & -1.089589 \\
$\mathrm{H}$ & -2.268823 & 0.675842 & 1.917570 \\
$\mathrm{H}$ & -1.832178 & -0.793625 & 2.287777 \\
$\mathrm{H}$ & 0.229261 & 2.473133 & 1.817687 \\
$\mathrm{H}$ & 0.216973 & 2.514918 & 0.220335 \\
$\mathrm{H}$ & 0.498645 & -2.824163 & 0.551856 \\
$\mathrm{H}$ & -0.339172 & -2.344305 & -0.689432 \\
& 1.471482 & 0.100167 & 3.126307 \\
$\mathrm{H}$ & 0.140686 & -0.950045 & 3.074080 \\
$\mathrm{H}$ & -1.253612 & 0.395883 & -1.755599 \\
$\mathrm{H}$ & & 3.493905 \\
\hline
\end{tabular}




$\begin{array}{rrrr}\mathrm{H} & -1.886963 & -1.360164 & 4.412159 \\ \mathrm{O} & 1.819701 & -0.244797 & -2.658395 \\ \mathrm{H} & 2.043922 & -1.102604 & -3.096988 \\ \mathrm{H} & 2.324796 & 0.474324 & -3.108298 \\ \mathrm{O} & -2.344454 & 0.493384 & -2.882292 \\ \mathrm{H} & -2.562854 & 1.463531 & -3.178084 \\ \mathrm{H} & -2.812397 & -0.136194 & -3.149351\end{array}$

$\left[\mathrm{Fe}\left(\mathrm{H}_{2} \mathrm{O}\right)_{6} \cdot 4^{\mathrm{H}_{2}} \mathrm{O}\right]^{2+} @ \mathrm{CB}[7]$

Atom $\mathrm{X}$

C $\quad-1.785241 \quad-4.580844$

$\mathrm{Z}$

$\begin{array}{llll}\mathrm{N} & -2.938814 & -4.503755 & 0.942517\end{array}$

$\begin{array}{llll}\text { C } & -2.788757 & -5.138225 & -0.359917\end{array}$

$\begin{array}{llll}\text { C } & -1.282477 & -5.590722 & -0.377517\end{array}$

$\begin{array}{llll}\mathrm{N} & -0.799491 & -5.187969 & 0.937562\end{array}$

$\begin{array}{llll}\mathrm{N} & -0.722735 & -4.863565 & -1.502437\end{array}$

$\begin{array}{llll}\text { C } & -1.685459 & -4.102813 & -2.181101\end{array}$

$\begin{array}{llll}\mathrm{N} & -2.889989 & -4.255305 & -1.501289\end{array}$

$\begin{array}{llll}\text { C } & -4.130300 & -3.739660 & -2.042602\end{array}$

$\begin{array}{llll}\mathrm{N} & -4.641185 & -2.550866 & -1.388987\end{array}$

$\begin{array}{llll}\text { C } & -5.435547 & -2.575270 & -0.173989\end{array}$

$\begin{array}{llll}\text { C } & -5.938195 & -1.094539 & -0.047328\end{array}$

N $\quad-5.454971 \quad-0.461164 \quad-1.259165$

C $\quad-4.713266 \quad-1.331292 \quad-2.050208$

$\begin{array}{llll}\mathrm{N} & -4.701276 & -2.796232 & 1.056329\end{array}$

C $\quad-4.707444 \quad-1.678128 \quad 1.890879$

$\begin{array}{llll}\mathrm{N} & -5.326806 & -0.642946 & 1.189068\end{array}$

$\begin{array}{llll}\text { C } & -5.922472 & 0.823179 & -1.751446\end{array}$

$\begin{array}{llll}\mathrm{N} & -5.234865 & 1.981329 & -1.215997\end{array}$

$\begin{array}{llll}\mathrm{C} & -5.482376 & 2.545557 & 0.095845\end{array}$

$\begin{array}{llll}\text { C } & -4.614991 & 3.856907 & 0.097225\end{array}$

$\begin{array}{llll}\mathrm{N} & -3.993809 & 3.853155 & -1.212306\end{array}$

$\begin{array}{llll}\text { C } & -4.405884 & 2.779477 & -2.000477\end{array}$

$\begin{array}{llll}\mathrm{N} & -4.947327 & 1.781102 & 1.211628\end{array}$

C $\quad-3.892013 \quad 2.427880 \quad 1.833664$

$\begin{array}{llll}\mathrm{N} & -3.704687 & 3.652751 & 1.211567\end{array}$

$\begin{array}{llll}\text { C } & -3.219636 & 4.949198 & -1.765766\end{array}$

$\begin{array}{llll}\mathrm{N} & -1.843213 & 5.041486 & -1.313393\end{array}$

$\begin{array}{llll}\text { C } & -1.414678 & 5.681924 & -0.095068\end{array}$

$\begin{array}{llll}\text { C } & 0.142733 & 5.747135 & -0.241476\end{array}$

$\begin{array}{llll}\mathrm{N} & 0.402713 & 4.826439 & -1.342988\end{array}$

$\begin{array}{llll}\text { C } & -0.778385 & 4.505477 & -2.041480\end{array}$

$\begin{array}{llll}\mathrm{N} & 0.622822 & 5.299024 & 1.050208\end{array}$

$\begin{array}{llll}\text { C } & -0.386055 & 4.709386 & 1.801281\end{array}$

$\begin{array}{llll}\mathrm{N} & -1.583775 & 4.884242 & 1.118817\end{array}$

$\begin{array}{llll}\text { C } & -5.615212 & 0.631195 & 1.807161\end{array}$

$\begin{array}{llll}\text { C } & -2.860007 & 4.681690 & 1.782829\end{array}$

$\begin{array}{llll}\text { O } & -4.109913 & 2.589029 & -3.170093\end{array}$

$\begin{array}{llll}\text { O } & -3.235243 & 2.000287 & 2.782539\end{array}$ 


\begin{tabular}{|c|c|c|c|}
\hline & & & \\
\hline & & & \\
\hline & & & \\
\hline & & & \\
\hline & 4.580526 & & \\
\hline & & & \\
\hline & & & \\
\hline & & & \\
\hline & & & \\
\hline & & & \\
\hline & & & \\
\hline & 48 & & \\
\hline & & & \\
\hline & & & \\
\hline & & & \\
\hline & & & \\
\hline & & & \\
\hline & & & \\
\hline & & & \\
\hline & & & \\
\hline & & & \\
\hline & & & \\
\hline & & & \\
\hline & & & \\
\hline & & & \\
\hline & & & \\
\hline & & & \\
\hline & & & \\
\hline & & & \\
\hline & & & \\
\hline & & & \\
\hline & & & \\
\hline & & & \\
\hline & & & \\
\hline & & & \\
\hline & & & \\
\hline & & & \\
\hline & & & \\
\hline & & & \\
\hline & & & \\
\hline & -4.2 & & \\
\hline & -4.2 & 686 & \\
\hline & & & \\
\hline & & & -3.1 \\
\hline & & & \\
\hline & -5.2( & & \\
\hline & & & \\
\hline & & 6.757500 & -0.4 \\
\hline & -1.894155 & 6.669574 & \\
\hline & 5.711640 & 3.987980 & -0.1829 \\
\hline
\end{tabular}




\begin{tabular}{|c|c|c|c|}
\hline & & & \\
\hline & & & \\
\hline & & & \\
\hline & & & \\
\hline & & & \\
\hline & & & \\
\hline & & & \\
\hline & & -6.5 & \\
\hline & & -5.2 & \\
\hline & & & \\
\hline & & & \\
\hline & -3.4 & & \\
\hline & -2.6 & & \\
\hline & & & \\
\hline & & & \\
\hline & & & \\
\hline & & & \\
\hline & & & \\
\hline & & & \\
\hline & -4.8 & & \\
\hline & & & \\
\hline & & & \\
\hline & & -2.8 & \\
\hline & & & \\
\hline & & & \\
\hline & & & \\
\hline & & & \\
\hline & -3.7 & & \\
\hline & & & \\
\hline & & & \\
\hline & & & \\
\hline & & & \\
\hline & & & \\
\hline & & & \\
\hline & -4.0 & -4.0 & \\
\hline & -1.1 & & \\
\hline & & & \\
\hline & & & \\
\hline & & & \\
\hline $\mathrm{O}$ & & -0.5 & \\
\hline & -0.2 & -2.1 & \\
\hline $\mathrm{O}$ & -0.3 & & \\
\hline O & & -0.3 & \\
\hline $\mathrm{O}$ & & & \\
\hline $\mathrm{U}$ & & & \\
\hline & 2.19 & -0.3 & \\
\hline & & & \\
\hline & & -0.82 & \\
\hline & & 2.497025 & \\
\hline & 0.254792 & 2.263681 & 0.40715 \\
\hline
\end{tabular}




$\begin{array}{crrr}\mathrm{H} & 0.441520 & -2.793127 & 0.338097 \\ \mathrm{H} & -0.312296 & -2.107602 & -0.847289 \\ \mathrm{H} & 1.431564 & -0.007181 & 3.428810 \\ \mathrm{H} & 0.122327 & -1.005856 & 3.455832 \\ \mathrm{H} & 0.392217 & 0.419434 & -1.664286 \\ \mathrm{H} & -1.152211 & 0.613421 & -1.624961 \\ \mathrm{O} & 2.425799 & 0.768902 & 4.274026 \\ \mathrm{H} & 3.264515 & 0.307146 & 4.050743 \\ \mathrm{H} & 2.579753 & 1.697325 & 3.994291 \\ \mathrm{O} & -1.418059 & -1.751132 & 3.922378 \\ \mathrm{H} & -1.532019 & -2.704221 & 3.670394 \\ \mathrm{H} & -2.086359 & -1.565909 & 4.604195 \\ \mathrm{O} & 1.839483 & -0.166274 & -2.574896 \\ \mathrm{H} & 1.993835 & -0.999280 & -3.075011 \\ \mathrm{H} & 2.320163 & 0.553716 & -3.046355 \\ \mathrm{O} & -2.042681 & 0.627979 & -2.925555 \\ \mathrm{H} & -2.386318 & 1.476025 & -3.272892 \\ \mathrm{H} & -2.736055 & -0.036735 & -3.147428\end{array}$

TABLE S7: Atomic Cartesian coordinates (in $\AA$ ) for inclusion compounds $\left[\mathrm{Fe}\left(\mathrm{H}_{2} \mathrm{O}\right)_{6} \cdot 4 \mathrm{H}_{2} \mathrm{O}\right]^{3+/ 2+} @ \mathrm{CB}[8]$ optimized at the PBE/TZVP level

\begin{tabular}{|c|c|c|c|}
\hline \multicolumn{4}{|c|}{$\left[\mathrm{Fe}\left(\mathrm{H}_{2} \mathrm{O}\right)_{6} \cdot 4 \mathrm{H}_{2} \mathrm{O}\right]^{3+} @ \mathrm{CB}[8]$} \\
\hline Aton & $\mathrm{n} \quad \mathrm{x}$ & $\mathrm{y}$ & $\mathrm{Z}$ \\
\hline $\mathrm{N}$ & 4.678424 & 3.853796 & 1.166307 \\
\hline $\mathrm{C}$ & 5.434953 & 3.900664 & -0.071416 \\
\hline $\mathrm{C}$ & 6.170219 & 2.516725 & -0.099403 \\
\hline $\mathrm{N}$ & 5.816852 & 1.911946 & 1.164276 \\
\hline $\mathrm{C}$ & 4.957814 & 2.710251 & 1.927044 \\
\hline $\mathrm{N}$ & 5.597770 & 1.854525 & -1.268583 \\
\hline $\mathrm{C}$ & 4.706903 & 2.674451 & -1.951168 \\
\hline $\mathrm{N}$ & 4.628260 & 3.878149 & -1.280900 \\
\hline $\mathrm{C}$ & 3.934861 & 5.033967 & -1.824654 \\
\hline $\mathrm{N}$ & 2.675430 & 5.350339 & -1.186054 \\
\hline $\mathrm{C}$ & 2.566881 & 6.133945 & 0.030597 \\
\hline $\mathrm{C}$ & 1.045809 & 6.506820 & 0.083667 \\
\hline $\mathrm{N}$ & 0.518218 & 5.995464 & -1.159517 \\
\hline $\mathrm{C}$ & 1.483384 & 5.333008 & -1.925582 \\
\hline $\mathrm{N}$ & 2.774889 & 5.388503 & 1.261315 \\
\hline $\mathrm{C}$ & 1.604395 & 5.187295 & 1.967222 \\
\hline $\mathrm{N}$ & 0.571246 & 5.811462 & 1.277672 \\
\hline $\mathrm{C}$ & -0.756529 & 6.395020 & -1.723418 \\
\hline $\mathrm{N}$ & -1.912285 & 5.733758 & -1.147922 \\
\hline $\mathrm{C}$ & -2.509974 & 6.108154 & 0.112725 \\
\hline $\mathrm{C}$ & -3.911055 & 5.404520 & 0.088920 \\
\hline $\mathrm{N}$ & -3.886819 & 4.653154 & -1.151238 \\
\hline $\mathrm{C}$ & -2.731173 & 4.892570 & -1.908537 \\
\hline $\mathrm{N}$ & -1.859631 & 5.527895 & 1.284918 \\
\hline $\mathrm{C}$ & -2.692523 & 4.647780 & 1.963815 \\
\hline $\mathrm{N}$ & -3.899499 & 4.592766 & 1.296477 \\
\hline
\end{tabular}




\begin{tabular}{|c|c|c|c|}
\hline & -5.061697 & & \\
\hline & -5.447017 & & \\
\hline & & & \\
\hline & & & \\
\hline & -5.894243 & & \\
\hline & 5.263078 & & \\
\hline & & & \\
\hline & -5.408773 & & \\
\hline & -6.0 & & \\
\hline & & & \\
\hline & & & \\
\hline & $-6.1^{\prime}$ & & \\
\hline & & & \\
\hline & & & \\
\hline & & & \\
\hline & -5.8 & & \\
\hline & -4.9 & & \\
\hline & & & \\
\hline & & & \\
\hline & -2.6 & & \\
\hline & & & \\
\hline & & & \\
\hline & & & \\
\hline & -1.4 & & \\
\hline & -2.7 & & \\
\hline & -1.6 & & \\
\hline & & & \\
\hline & & & \\
\hline & & & \\
\hline & & & \\
\hline & & & \\
\hline & & & \\
\hline & & & \\
\hline & & & \\
\hline & & & \\
\hline & & & \\
\hline & & & \\
\hline & & & \\
\hline & & & \\
\hline & & & \\
\hline & & & \\
\hline & & & \\
\hline & & & \\
\hline & & & \\
\hline & & & \\
\hline & & & \\
\hline & & & \\
\hline & & & \\
\hline & 6.210901 & 0.695737 & -1.89315 \\
\hline & 4.656659 & -1.489164 & -3.0216 \\
\hline
\end{tabular}




\begin{tabular}{|c|c|c|c|}
\hline & 2.496572 & -4.456817 & \\
\hline & 2.406445 & -4.032956 & 2.994197 \\
\hline & 0.695110 & & \\
\hline & -1.328335 & & \\
\hline & .495250 & -4.569066 & \\
\hline & & & \\
\hline ) & -4.102538 & -2.382277 & \\
\hline & -4.544537 & -2.465343 & \\
\hline & & -0.758960 & \\
\hline ) & & & \\
\hline ) & -4.9 & & \\
\hline & -5.063086 & & \\
\hline & -2.4 & 4.461994 & \\
\hline D & -2.4 & & \\
\hline 4 & -0.6 & & \\
\hline & & & \\
\hline ) & & & \\
\hline C & & & \\
\hline D & 4.0 & & \\
\hline $\mathrm{O}$ & 4.5 & & \\
\hline $\mathrm{O}$ & -0.2 & & \\
\hline $\mathrm{O}$ & & -0.0 & \\
\hline & 0.0 & -0.0 & -0 . \\
\hline ) & 0.0 & -0.0 & \\
\hline D & & & \\
\hline $\mathrm{O}$ & -0.1 & & \\
\hline $\mathrm{O}$ & -2.0 & 0.1 & -0.2 \\
\hline $\mathrm{O}$ & $2.0^{\prime}$ & & \\
\hline D & -2.5 & -0 . & \\
\hline D & 2.5 & & -2.7 \\
\hline $\mathrm{O}$ & 0.2 & -2.5 & \\
\hline $\mathrm{H}$ & -7.2 & -2.5 & \\
\hline I & -6.1 & -4.7 & \\
\hline $\mathrm{H}$ & -7.0 & & \\
\hline I & -7.6 & 0.8 & -0 . \\
\hline $\mathrm{H}$ & -2.56 & 7.2 & \\
\hline $\mathrm{H}$ & -4.76 & & \\
\hline $\mathrm{H}$ & & & -0.0 \\
\hline$H$ & 0.8 & 7.5 & \\
\hline $\mathrm{H}$ & 7.26 & 2.59 & -0.2 \\
\hline $\mathrm{H}$ & 6.11 & 4.76 & -0.0 \\
\hline $\mathrm{H}$ & -3.2 & -7.0 & \\
\hline $\mathrm{H}$ & -0.85 & -7.589760 & \\
\hline $\mathrm{H}$ & 7.58 & 0.879845 & \\
\hline $\mathrm{H}$ & & & \\
\hline $\mathrm{H}$ & -4.75 & & \\
\hline $\mathrm{H}$ & -3.943965 & -4.781010 & \\
\hline $\mathrm{H}$ & -7.582241 & -0.879132 & 1.60245 \\
\hline $\mathrm{H}$ & -6.245277 & -0.737508 & \\
\hline & -5.923548 & 4.605524 & 1.778687 \\
\hline
\end{tabular}




\begin{tabular}{crrr}
$\mathrm{H}$ & -4.846821 & 3.690269 & 2.894758 \\
$\mathrm{H}$ & -0.825912 & 7.224342 & 1.919435 \\
$\mathrm{H}$ & -0.647497 & 5.765875 & 2.948400 \\
$\mathrm{H}$ & 4.753997 & 5.884020 & 1.675984 \\
$\mathrm{H}$ & 3.946101 & 4.783588 & 2.850387 \\
$\mathrm{H}$ & 0.871479 & -7.481559 & -1.616539 \\
$\mathrm{H}$ & 0.733504 & -6.122400 & -2.791933 \\
$\mathrm{H}$ & 4.612508 & 5.903031 & -1.760979 \\
$\mathrm{H}$ & 3.712513 & 4.814874 & -2.878744 \\
$\mathrm{H}$ & -0.873597 & 7.487437 & -1.612643 \\
$\mathrm{H}$ & -0.734340 & 6.130870 & -2.790824 \\
$\mathrm{H}$ & -5.917327 & 4.758751 & -1.649455 \\
$\mathrm{H}$ & -4.833760 & 3.940644 & -2.832774 \\
$\mathrm{H}$ & -7.308251 & -0.831459 & -1.890800 \\
$\mathrm{H}$ & -5.859305 & -0.647486 & -2.932946 \\
$\mathrm{H}$ & -4.615422 & -5.903362 & -1.759849 \\
$\mathrm{H}$ & -3.716673 & -4.816054 & -2.879433 \\
$\mathrm{H}$ & 4.837550 & -3.942318 & -2.832478 \\
$\mathrm{H}$ & 5.918916 & -4.760456 & -1.647271 \\
$\mathrm{H}$ & 0.826386 & -7.228814 & 1.915671 \\
$\mathrm{H}$ & 0.647963 & -5.772924 & 2.948334 \\
$\mathrm{H}$ & 7.307149 & 0.832851 & -1.890379 \\
$\mathrm{H}$ & 5.858430 & 0.647796 & -2.932596 \\
$\mathrm{H}$ & -1.081152 & 2.977639 & 2.928057 \\
$\mathrm{H}$ & 1.081996 & -2.981231 & 2.931259 \\
$\mathrm{H}$ & -0.489518 & -3.156964 & 2.999361 \\
$\mathrm{H}$ & 4.842757 & -4.602029 & 1.780294 \\
$\mathrm{H}$ & 4.762831 & -6.104596 & 0.110317 \\
$\mathrm{H}$ & 2.566907 & -7.205370 & 0.209909 \\
$\mathrm{H}$ & 7.672154 & -0.881758 & -0.164308 \\
$\mathrm{H}$ & 7.054419 & -3.251663 & 0.032065 \\
$\mathrm{H}$ & -0.299816 & 2.627850 & -0.507930 \\
$\mathrm{H}$ & -0.041161 & 2.412105 & 1.098465 \\
$\mathrm{H}$ & 2.402786 & 0.026641 & -1.216396 \\
$\mathrm{H}$ & 2.603108 & 0.350438 & 0.385934 \\
$\mathrm{H}$ & 0.304809 & -2.632533 & -0.504793 \\
$\mathrm{H}$ & 0.043103 & -2.415902 & 1.100673 \\
$\mathrm{H}$ & -2.399759 & -0.032157 & -1.216286 \\
$\mathrm{H}$ & -2.599447 & -0.357481 & 0.385630 \\
\hline
\end{tabular}




\begin{tabular}{crrr}
{$\left[\mathbf{F e}\left(\mathbf{H}_{\mathbf{2}} \mathbf{O}\right)_{6} \cdot \mathbf{4 H} \mathbf{H}_{\mathbf{2}} \mathbf{O}\right]^{2+} \mathbf{C C B}[\mathbf{8}]$} & \\
$\mathrm{Atom}$ & $\mathrm{X}$ & $\mathrm{y}$ & $\mathrm{Z}$ \\
$\mathrm{N}$ & -1.847694 & -5.818049 & 1.166237 \\
$\mathrm{C}$ & -2.483466 & -6.166458 & -0.083982 \\
$\mathrm{C}$ & -3.843712 & -5.388723 & -0.032940 \\
$\mathrm{~N}$ & -3.764505 & -4.641578 & 1.205468 \\
$\mathrm{C}$ & -2.620931 & -4.956501 & 1.951573 \\
$\mathrm{~N}$ & -3.820044 & -4.581518 & -1.241220 \\
$\mathrm{C}$ & -2.643576 & -4.741253 & -1.963261 \\
$\mathrm{~N}$ & -1.842923 & -5.641612 & -1.281578 \\
$\mathrm{C}$ & -0.669328 & -6.239272 & -1.892123 \\
$\mathrm{~N}$ & 0.589519 & -5.890304 & -1.274465 \\
$\mathrm{C}$ & 1.116630 & -6.551326 & -0.097059 \\
$\mathrm{C}$ & 2.611345 & -6.080081 & -0.056422 \\
$\mathrm{~N}$ & 2.754858 & -5.279820 & -1.251681 \\
$\mathrm{C}$ & 1.574076 & -5.207493 & -1.999339 \\
$\mathrm{~N}$ & 0.582983 & -6.093723 & 1.172735 \\
$\mathrm{C}$ & 1.516913 & -5.407364 & 1.932449 \\
$\mathrm{~N}$ & 2.697856 & -5.345151 & 1.197727 \\
$\mathrm{C}$ & 4.033199 & -4.906919 & -1.820930 \\
$\mathrm{~N}$ & 4.663911 & -3.760146 & -1.198076 \\
$\mathrm{C}$ & 5.407672 & -3.838038 & 0.042092 \\
$\mathrm{C}$ & 6.191276 & -2.480633 & 0.090285 \\
$\mathrm{~N}$ & 5.836977 & -1.841357 & -1.156838 \\
$\mathrm{C}$ & 4.976239 & -2.615897 & -1.943029 \\
$\mathrm{~N}$ & 4.598971 & -3.807136 & 1.249691 \\
$\mathrm{C}$ & 4.770139 & -2.633628 & 1.973395 \\
$\mathrm{~N}$ & 5.676992 & -1.840187 & 1.291743 \\
$\mathrm{C}$ & 6.524711 & -0.693964 & -1.704362 \\
$\mathrm{~N}$ & 6.114473 & 0.589332 & -1.165673 \\
$\mathrm{C}$ & 6.571308 & 1.124805 & 0.104070 \\
$\mathrm{C}$ & 6.092011 & 2.616852 & 0.064863 \\
$\mathrm{C}$ & 0.671113 & 6.271651 & -1.891720 \\
$\mathrm{~N}$ & -0.586969 & 5.912282 & -1.277710 \\
$\mathrm{C}$ & -1.119943 & 6.566965 & -0.099733 \\
$\mathrm{~N}$ & 5.356562 & 2.700903 & -1.189232 \\
$\mathrm{C}$ & 5.421311 & 1.519278 & -1.922449 \\
$\mathrm{~N}$ & 5.914446 & 0.592946 & 1.281483 \\
$\mathrm{C}$ & 5.225710 & 1.572587 & 2.006980 \\
$\mathrm{~N}$ & 5.290459 & 2.754461 & 1.259766 \\
$\mathrm{C}$ & 4.988294 & 3.960223 & -1.804004 \\
$\mathrm{~N}$ & 3.814874 & 4.599221 & -1.242597 \\
$\mathrm{C}$ & 3.843180 & 5.404921 & -0.033076 \\
$\mathrm{~N}$ & 2.485744 & 6.188315 & -0.081932 \\
$\mathrm{C}$ & 1.846512 & 5.674914 & -1.284719 \\
$\mathrm{C}$ & 3.642228 & 4.771172 & -1.967467 \\
& 1.845321 & 4.656889 & 1.204661 \\
& 4.966586 & 1.947514 \\
\hline
\end{tabular}




\begin{tabular}{|c|c|c|c|}
\hline & -2.612219 & 6.087646 & \\
\hline & & 5.280095 & \\
\hline & -1.563452 & 5.216759 & \\
\hline & -0.585439 & & \\
\hline & & & \\
\hline & -2.699906 & & \\
\hline & -4.020077 & & \\
\hline & -4.651043 & 3.755353 & \\
\hline & -5.40 & & \\
\hline & -6.13 & & \\
\hline & -5.8 & & \\
\hline & $-4.9^{\prime}$ & 2.6 & -1.9 \\
\hline & -4.6 & 3.8 & \\
\hline & -4.7 & & \\
\hline & -5.6 & 605 & \\
\hline & -6.5 & 093 & -1.7 \\
\hline & -6.1 & & \\
\hline & -6.5 & & \\
\hline & -6.1 & -2.6060 & \\
\hline & -5.3 & -2.6 & \\
\hline 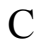 & -5.4 & -1.5 & -1.9 \\
\hline & -5.9 & & \\
\hline & -5.2 & -1.56 & \\
\hline & -5.2 & -2.7 & \\
\hline 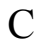 & -4.9 & -4.0 & \\
\hline & -6.2 & $0.6^{\prime}$ & \\
\hline & -4.6 & -1.4 & \\
\hline & -4.9 & -3.9 & \\
\hline O & -4.9 & -1.3 & \\
\hline 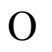 & -4.5 & 2.3 & -3. \\
\hline O & -4.2 & & \\
\hline & -3.9 & 4.9 & \\
\hline D & -1.4 & & -3.0 \\
\hline & -1.3 & & \\
\hline & & 6.5 & \\
\hline o & & 4.22 & -3.0 \\
\hline $\mathrm{O}$ & 2.35 & 4.57 & \\
\hline & 4.91 & 4.02 & \\
\hline $\mathrm{O}$ & & & -3.0 \\
\hline $\mathrm{O}$ & 4.65 & 1.429647 & \\
\hline & 6.27 & -0.66 & \\
\hline O & & -2.3 & -3.0 \\
\hline $\mathrm{O}$ & 4.22 & -2.356357 & \\
\hline $\mathrm{C}$ & 3.95 & -4.97 & \\
\hline $\mathrm{O}$ & & -4.6 & \\
\hline $\mathrm{O}$ & & -4.945624 & \\
\hline $\mathrm{C}$ & -0.698540 & -6.505202 & 1.712191 \\
\hline $\mathrm{O}$ & -2.370268 & -4.192716 & -3.02973 \\
\hline $\mathrm{O}$ & -2.36 & & \\
\hline $\mathrm{O}$ & 1.492979 & -2.169339 & 2.770365 \\
\hline
\end{tabular}




\begin{tabular}{|c|c|c|c|}
\hline $\mathrm{O}$ & -0.005637 & 0.000342 & 2.093833 \\
\hline $\mathrm{Fe}$ & 0.004193 & -0.041402 & 0.012932 \\
\hline $\mathrm{O}$ & 0.175637 & -0.255750 & -2.166057 \\
\hline $\mathrm{O}$ & 0.743759 & -2.085819 & 0.162679 \\
\hline $\mathrm{O}$ & -0.809694 & 1.998019 & 0.099930 \\
\hline $\mathrm{O}$ & 1.979654 & 0.790483 & -0.184944 \\
\hline $\mathrm{O}$ & -1.990613 & -0.815874 & -0.221519 \\
\hline $\mathrm{O}$ & 2.195655 & 1.498658 & -2.756954 \\
\hline $\mathrm{O}$ & -2.220131 & -1.455342 & -2.800468 \\
\hline $\mathrm{O}$ & -1.487443 & 2.179369 & 2.731220 \\
\hline $\mathrm{H}$ & 4.732851 & 6.060363 & -0.036396 \\
\hline $\mathrm{H}$ & 2.607205 & 7.284028 & -0.156709 \\
\hline $\mathrm{H}$ & 7.667616 & 1.013498 & 0.188579 \\
\hline $\mathrm{H}$ & 6.921301 & 3.347496 & 0.067451 \\
\hline $\mathrm{H}$ & 6.062977 & -4.727858 & 0.049456 \\
\hline $\mathrm{H}$ & 7.286907 & -2.602466 & 0.166576 \\
\hline $\mathrm{H}$ & 0.999875 & -7.647025 & -0.183332 \\
\hline $\mathrm{H}$ & 3.337487 & -6.913356 & -0.058354 \\
\hline $\mathrm{H}$ & -4.730812 & -6.047667 & -0.036129 \\
\hline $\mathrm{H}$ & -2.600625 & -7.262017 & -0.167872 \\
\hline $\mathrm{H}$ & -1.008437 & 7.663399 & -0.182185 \\
\hline $\mathrm{H}$ & -3.342951 & 6.916803 & -0.071632 \\
\hline $\mathrm{H}$ & -5.776794 & -4.709535 & 1.779461 \\
\hline $\mathrm{H}$ & -4.645382 & -3.836958 & 2.881886 \\
\hline $\mathrm{H}$ & 0.813479 & 7.604597 & 1.552454 \\
\hline $\mathrm{H}$ & 0.674384 & 6.297932 & 2.787755 \\
\hline $\mathrm{H}$ & 5.774327 & 4.718421 & 1.779683 \\
\hline $\mathrm{H}$ & 4.640591 & 3.847259 & 2.880747 \\
\hline $\mathrm{H}$ & 7.372878 & -0.779774 & 1.879577 \\
\hline $\mathrm{H}$ & 5.920769 & -0.623580 & 2.938152 \\
\hline $\mathrm{H}$ & 4.657593 & -5.831341 & 1.733554 \\
\hline $\mathrm{H}$ & 3.754450 & -4.769744 & 2.871278 \\
\hline $\mathrm{H}$ & -0.816500 & -7.591524 & 1.551524 \\
\hline $\mathrm{H}$ & -0.678108 & -6.287714 & 2.789845 \\
\hline $\mathrm{H}$ & -4.707568 & 5.764007 & -1.783916 \\
\hline $\mathrm{H}$ & -3.834893 & 4.622330 & -2.875413 \\
\hline $\mathrm{H}$ & -0.790357 & -7.338145 & -1.881926 \\
\hline $\mathrm{H}$ & -0.626370 & -5.879585 & -2.930432 \\
\hline $\mathrm{H}$ & 4.717356 & -5.773913 & -1.766980 \\
\hline $\mathrm{H}$ & 3.853802 & -4.638631 & -2.872689 \\
\hline $\mathrm{H}$ & 7.611177 & -0.811302 & -1.544468 \\
\hline $\mathrm{H}$ & 6.306123 & -0.674093 & -2.781827 \\
\hline $\mathrm{H}$ & 5.839307 & 4.661119 & -1.725275 \\
\hline $\mathrm{H}$ & 4.780189 & 3.756322 & -2.863624 \\
\hline $\mathrm{H}$ & 0.786094 & 7.370871 & -1.873217 \\
\hline $\mathrm{H}$ & 0.631891 & 5.919276 & -2.932627 \\
\hline $\mathrm{H}$ & -6.328145 & 0.682331 & -2.779412 \\
\hline $\mathrm{H}$ & -7.625816 & 0.825130 & -1.535307 \\
\hline $\mathrm{H}$ & -4.658607 & 5.845574 & 1.730358 \\
\hline $\mathrm{H}$ & -3.755327 & 4.784756 & 2.868634 \\
\hline
\end{tabular}




$\begin{array}{rrrr}\mathrm{H} & -5.844362 & -4.653474 & -1.721258 \\ \mathrm{H} & -4.791716 & -3.744568 & -2.862102 \\ \mathrm{H} & -7.370600 & 0.794718 & 1.884940 \\ \mathrm{H} & -5.914935 & 0.635810 & 2.938241 \\ \mathrm{H} & -7.287945 & 2.618988 & 0.173223 \\ \mathrm{H} & -6.055476 & 4.739022 & 0.033608 \\ \mathrm{H} & -6.931275 & -3.336211 & 0.072750 \\ \mathrm{H} & -7.676048 & -1.002262 & 0.199046 \\ \mathrm{H} & 1.394535 & -2.280759 & -0.534948 \\ \mathrm{H} & 1.178311 & -2.210299 & 1.059442 \\ \mathrm{H} & -2.139622 & -1.148553 & -1.169451 \\ \mathrm{H} & -2.158958 & -1.564271 & 0.379232 \\ \mathrm{H} & -1.524166 & 2.177573 & -0.536049 \\ \mathrm{H} & -1.179320 & 2.147580 & 1.023359 \\ \mathrm{H} & 2.123286 & 1.159416 & -1.118430 \\ \mathrm{H} & 2.136774 & 1.515673 & 0.445958 \\ \mathrm{H} & 0.757342 & 0.351187 & -2.684570 \\ \mathrm{H} & -0.467622 & 0.688403 & 2.636721 \\ \mathrm{H} & -0.603046 & -0.536821 & -2.702319 \\ \mathrm{H} & 0.458446 & -0.681844 & 2.642412 \\ \mathrm{H} & -2.152574 & -2.420682 & -3.024881 \\ \mathrm{H} & -3.117346 & -1.199858 & -3.126515 \\ \mathrm{H} & 3.095105 & 1.250111 & -3.085250 \\ \mathrm{H} & 2.114196 & 2.458801 & -2.995716 \\ \mathrm{H} & 2.453625 & -2.098669 & 3.009520 \\ \mathrm{H} & 1.229693 & -3.055670 & 3.117381 \\ \mathrm{H} & -2.445741 & 2.106963 & 2.979568 \\ \mathrm{H} & -1.224977 & 3.068959 & 3.072145\end{array}$

